\title{
LOGÍSTICA HUMANITÁRIA: UMA ANALISE SOBRE O ATENDIMENTO ÀS VÍTIMAS DO DESASTRE NATURAL DA REGIÃO SERRANA FLUMINENSE EM 2011
}

\author{
José Itamar Monteiro ${ }^{1}$ \\ Daniel Bertoli Gonçalves ${ }^{2}$ \\ Marina Martins Moises ${ }^{3}$
}

\author{
${ }^{1}$ Mestrando no Programa de Pos-Graduação em Processos Tecnológicos e Ambientais - UNISO. \\ Professor da FATEC-Sorocaba-SP.jitam@ bol.com.br \\ ${ }^{2}$ Professor e Pesquisador do Programa de Pos-Graduação em Processos Tecnológicos e Ambientais - Mestrado Profissional. \\ danielbertoli@bol.com.br \\ ${ }^{3}$ Tecnóloga em Logística. marinamartins_m@hotmail.com
}

Recebido em: 27/05/2014 - Aprovado em: 15/09/2014 - Disponibilizado em: 15/12/2014

\begin{abstract}
RESUMO: Nas últimas décadas, temos observado um aumento significativo do número de vítimas de desastres naturais, tais como terremotos, enchentes, tsunamis, deslizamentos de encostas, furacões, entre outras situações. Após tais catástrofes, os sobreviventes acabam por se encontrar em meio a grandes dificuldades como a falta de mantimentos, remédios, materiais de higiene pessoal, roupas, calçados, cobertores e principalmente abrigo. Felizmente, a ajuda às vitimas por parte do Estado, de ONG's, empresas e até mesmo por voluntários, tem sido eficaz, graças principalmente a uma organização rápida com uso efetivo da logística em seus eixos emblemáticos, eficientes e com qualidade, para atender todas as vítimas, que tem sido conhecida como logística humanitária. O presente artigo discute este conceito a partir de uma abordagem teórica e prática, ressaltando a importância de sua efetiva aplicação em situações de desastre natural. Para isso, além de uma discussão conceitual a luz da bibliografia disponível, é apresentado um estudo de caso sobre a resposta imediata ás enchentes ocorridas na região serrana Fluminense em Janeiro de 2011, com especial enfoque na atuação do Exercito Brasileiro.
\end{abstract}

Palavras-chave: Logística Humanitária. Desastres Naturais. Otimização. Emergências. Suprimentos.

\section{Humanitarian Logistics: an analysis on the care to the victims of the natural disaster in mountainous area Fluminense in 2011}

\begin{abstract}
In recent decades we have witnessed a significant increase in the number of victims of natural disasters, such as earthquakes, floods, tsunamis, landslides, hurricanes, among other situations. After such disasters, the survivors eventually find yourself in the midst of great difficulties such as lack of food, medicine, toiletries, clothes, shoes, blankets and shelter materials mainly. Fortunately, help victims by the state, NGOs, companies and even by volunteers, has been effective, thanks mainly to rapid organization with effective use of logistics in their flagship, axes efficient and quality to meet all victims, which has been known as a humanitarian logistics. This article discusses this concept from a theoretical and practical approach, emphasizing the importance of their effective application in situations of natural disasters. For that, beyond a conceptual discussion the light of available literature, a case study on the immediate response to the floods occurred in the mountainous region of the Rio de Janeiro in January 2011, with special focus on the performance of the Brazilian Army is presented.
\end{abstract}

Keywords : Humanitarian Logistics. Natural Disasters. Optimization. Emergencies. Supplies.

\section{Introdução}

Terremotos, furacões, enchentes, maremotos e outras situações de natureza emergencial têm exigido um tratamento logístico especial que vem sendo denominado de logística humanitária, um conceito ainda muito novo no Brasil. 
Até 2006, existia um conjunto limitado de pesquisa sobre Logística Humanitária, e desde então diversos estudos vêm sendo desenvolvidos mostrando a importância desta ferramenta para as nações. (BEAMOM e KOTLEBA, 2006).

Ertem et al. (2010) destacam que, nas primeiras 24 horas após um desastre, profissionais de organizações humanitárias se deslocam para o local do evento com intuito de estimar as necessidades e, ainda nas primeiras 36 horas, os recursos costumam ser liberados para as organizações humanitárias e governos. Tais recursos são definidos pelo tipo e quantidade de material necessário, sendo que doações em espécie devem ser classificadas e contabilizadas, enquanto doações em dinheiro são destinadas às compras de suprimentos.

Dos desastres naturais que ocorrem no Brasil, 58\% são enchentes e $11 \%$ são deslizamentos (THENÓRIO, 2011).

Nas três últimas décadas, a taxa de catástrofes elevou-se de 50 para 400 por ano, de acordo com alguns autores como Kovacs e Spens (2009) e Gonçalves (2008), boa parte destes desastres naturais está associada ao aquecimento global, um fenômeno diretamente ligado a ação danosa do ser humano sobre o meio ambiente, como na emissão de poluentes atmosféricos, desmatamentos das áreas naturais, queimadas, entre outras.
Nos próximos 50 anos, prevê-se que tal taxa ainda aumente cinco vezes (Thomas e Kopczack, 2007). Em 2008, cerca de 200 milhões de pessoas sofreram com catástrofes, que causaram 240 mil mortes e prejuízos de 230 bilhões de dólares (BLECKEN, 2010).

Enchentes, deslizamentos, furacões, tsunamis estão se tornando cada vez mais comuns em todo o mundo, e seus danos poderiam ser minimizados se certas precauções fossem tomadas com antecedência.

No Brasil, a ajuda às vitimas por parte do Estado, de ONG's, empresas e até mesmo por voluntários, tem sido eficaz, graças a uma organização rápida com uso efetivo da logística humanitária em seus eixos emblemáticos, eficientes e com qualidade, para atender todas as vítimas. O presente artigo discute este conceito a partir de uma abordagem teórica e prática, ressaltando a importância de sua efetiva aplicação em situações de desastre natural.

Para isso, além de uma discussão conceitual a luz da bibliografia disponível, é apresentado um estudo de caso sobre a resposta imediata ás enchentes ocorridas na região serrana Fluminense em Janeiro de 2011, com especial enfoque na atuação do Exercito Brasileiro.

A análise é estruturada a partir dos fatores propostos por Martinez et al. (2010): acesso e logística; saúde; água, saneamento e higiene; 
alimentos; e abrigos e suprimentos não relacionados à alimentação.

\section{Logística Humanitária: principais conceitos e aplicações}

A logística humanitária é a função que visa o fluxo de pessoas e materiais de forma adequada e em tempo oportuno na cadeia de assistência, com o objetivo principal de atender de maneira correta o maior número de pessoas. (BEAMON e KOTLEBA, 2006).

Logística humanitária engloba, além de planejamento, suprimento, transporte, armazenamento, rastreamento, monitoramento e desembaraço alfandegário em resposta a catástrofes (KOVACS e SPENS, 2009 )

De acordo com TRB (2010), o conceito de ciclo de desastre implica em um processo contínuo em que o governo, as comunidades, as empresas e as pessoas devem fazer um planejamento para reduzir as perdas em caso de catástrofes. O ciclo de desastre é desencadeado por um evento e começa com a resposta a esse evento. A meta principal é responder a este evento com corretas especificações, de modo que a perda de vidas e bens seja minimizada e, posteriormente, reconstruir a região atingida de modo que se reduzam as perdas futuras. Os quatro componentes do ciclo de desastres são: Preparar, responder, recuperar e mitigar. i) Preparar: Se refere à preparação atividades, programas e sistemas desenvolvidos antes de um provável desastre. É uma etapa projetada para construir e reforçar as capacidades de indivíduos, empresas, comunidades e governos municipal, estadual e federal para apoio à resposta a e recuperação de futuro desastres.

ii) Responder: A resposta começa assim que um caso de desastres ocorre. A resposta é a prestação de busca e serviços de emergência médica serviços e controle de acesso bem como a reparação comunicação e restauração e sistemas de dados durante uma crise. Um plano coordenado de resposta pode ajudar a reduzir acidentes e danos, bem como diminuir o tempo de recuperação,

iii) Recuperar: As operações de recuperação têm o objetivo de fornecer o atendimento das necessidades básicas dos atingidos e a restauração dos sistemas comunitários. Há dois componentes fundamentais na fase de recuperação: durante a primeira fase, a infraestrutura é examinada e as reparações são efetuadas para restaurar o fornecimento de água, energia, comunicação, e outras necessidades. A segunda fase inclui a retorno às funções normais da região sinistrada e a tomada de providências para que sejam evitados desastres futuros.

iv) Mitigar: são providencias de curto e longo prazo com a meta de reduzir ou, até 
eliminar, as perdas de vidas e bens no futuro. As estratégias podem variar no tempo e no volume de recursos aplicados, mas sempre tem o objetivo de reduzir a vulnerabilidade e a exposição ao risco às populações em caso de prováveis catástrofes que certamente voltarão a ocorrer.

Por causa desse foco, os programas de gestão de emergências geralmente priorizam as fases preparação e resposta, deixando poucos recursos para atender as fases de mitigação das consequências dos desastres e a recuperação das áreas atingidas.

Conforme Fernandes (2010), de modo semelhante à divisão anterior, a Política Nacional de Defesa Civil do Brasil, prevê o gerenciamento de desastres também em quatro fases:

i) Prevenção de desastres: É considerada pela defesa civil como a etapa mais nobre de todo o processo, por ser menos dispendiosa e principalmente por ser a fase que permite maior redução de perdas de vidas. Esta fase compreende: avaliação de riscos de desastres e redução dos mesmos.

ii) Preparação e Alerta para Desastres: Tem por objetivo o desenvolvimento de projetos que proporcionem um aumento da capacidade de atendimento à emergência. Envolve: monitoramento, alarme, planejamento operacional e de contingência, mobilização e apoio logístico. iii) Atendimento à emergência: É a fase do atendimento propriamente dito. É a que demanda maior urgência abrangendo: $\mathrm{O}$ socorro às vítimas, a assistência à população vitimada e a avaliação dos danos.

iv) Reconstrução: Tem como objetivo o completo restabelecimento das condições de normalidade dos serviços públicos, da economia da região, do bem estar da população atingida.

\section{Logística Humanitária no Desastre climático na região serrana fluminense}

A tragédia climática ocorrida em janeiro de 2011 no estado do Rio de Janeiro afetou diretamente 20 municípios e 90 mil pessoas. Foram 30 mil desabrigados e desalojados, bem como 916 vítimas fatais de enchentes, deslizamentos e desabamentos ocorridos entre 11 e 12 de janeiro de 2011. Este desastre foi considerado na época o maior da história do país e o décimo pior deslizamento do mundo na última década $(\mathrm{O}$ EXÉRCITO, 2011). A cidade com mais vítimas da tragédia foi Nova Friburgo, seguida por Teresópolis, Petrópolis, Sumidouro, São José do Vale do Rio Preto e Bom Jardim.

Foram apontadas como causas do desastre a geologia da região, a ocupação 
irregular do solo (em encostas e áreas de várzea) e as chuvas de grande intensidade concentradas em períodos de 15 minutos. Contudo, observa-se um histórico de calamidades que ocorrem no Estado do Rio de Janeiro todos os anos, entre os meses de novembro e abril, devido a estes mesmos motivos. Logo, fica evidente o baixo investimento em prevenção e mitigação de desastres naquele estado. Em 2010, o valor investido ( $\mathrm{R} \$ 80$ milhões) para $\mathrm{a}$ reconstrução de locais atingidos pela chuva foi dez vezes superior ao valor aplicado $(\mathrm{R} \$$ 8 milhões) para a prevenção de catástrofes (CAMPANATO, 2011).

\subsection{Análise da operação humanitária}

Os atores envolvidos na operação humanitária em resposta ao desastre das enchentes na região serrana fluminense foram: o governo do estado, as prefeituras das cidades atingidas, a Defesa Civil do Estado do Rio de Janeiro, a Força Nacional de Segurança, o Corpo de Bombeiros Militar do Estado do Rio de Janeiro, a Marinha e a Aeronáutica e Exército Brasileiro, além de ONGs. Estes órgãos de apoio foram gerenciados pelo Centro de Gerenciamento de Crises, entidade do Sistema Nacional de Defesa Civil.

A participação das Forças Armadas na operação humanitária se deu por decisão do ministro chefe do Gabinete de Segurança
Institucional. A missão do Exército Brasileiro na fase de resposta ao evento consistia em apoiar os órgãos de Defesa Civil dos municípios afetados, executando tarefas logísticas, como: distribuição de donativos, transporte de desabrigados, evacuação de feridos, tratamento e distribuição de água em locais de difícil acesso, remoção de carros inundados (desobstrução de vias), abastecimento de combustível (viaturas), assistência religiosa.

\subsection{Acesso e logística}

$\mathrm{O}$ acesso à região serrana do Estado do Rio de Janeiro se dá a partir do transporte rodoviário, sendo as principais vias de acesso a BR 040, BR 116, BR 101 e RJ-116. Devido aos deslizamentos, alguns trechos destas vias ficaram bloqueados, sendo liberados poucos dias após a enchente. Porém, diversas vias no interior das cidades foram obstruídas devido a quedas de barreiras, que isolaram comunidades. Segundo dados levantados, um mês após o desastre, o acesso a todas as áreas onde houve vítimas já estava restabelecido. Contudo, três meses após as enchentes, ainda havia entulhos pelas ruas em Teresópolis (SOUTO, 2011).

O papel do Exército Brasileiro destacou-se na desobstrução de vias interditadas por meio do emprego de viaturas especializadas dotadas de ganchos. Esta ação foi essencial para possibilitar o deslocamento dos meios de 
socorro. Afinal, nas primeiras setenta e duas horas após o desastre, o Corpo de Bombeiros enfrentava grandes dificuldades para a realização de buscas e salvamentos. As tropas do EB também atuaram na realização de buscas e salvamentos, além de participar na recuperação da mobilidade da população por meio dos trabalhos de lançamentos de pontes. Foram montadas duas pontes, uma sobre o Rio Grande, na cidade de Bom Jardim, e outra sobre o Rio Paquequer, em Sumidouro, permitindo a ligação de áreas isoladas e a circulação normal de linhas de ônibus municipais e intermunicipais nestes locais.

O objetivo principal da operação de resposta imediata consistia em possibilitar o acesso à população local para a realização de salvamentos e para a distribuição de suprimentos emergenciais. $\mathrm{O}$ Exército Brasileiro também desempenhou a distribuição destes suprimentos, além do transporte de desabrigados/desalojados e da evacuação de feridos. Foi observada a necessidade do controle de tráfego devido às restrições de acesso e a grande demanda pelo serviço de distribuição resultante do grande volume de doações para atender a demanda das vítimas. $\mathrm{O}$ transporte das doações de outras regiões do Brasil para o Rio de Janeiro foi realizada pela Força Aérea Brasileira (FAB), que disponibilizou aeronaves, caminhões e carretas. Também foi imprescindível a utilização de helicópteros do Comando de Aviação do Exército para o transporte aéreo de suprimentos e para o acesso às regiões mais remotas. $\mathrm{O}$ Exército contou com o apoio de 42 viaturas e quatro helicópteros durante a fase de resposta imediata da operação humanitária.

Uma das dificuldades enfrentadas foi a restrição de veículos e recursos humanos para atender tanto ao transporte de gêneros alimentícios e medicamentos quanto ao transporte de desabrigados e feridos. Portanto, uma decisão crítica a ser tomada na operação se refere ao planejamento eficiente do uso dos recursos disponíveis para realizar o transporte de suprimentos e o salvamento de feridos e desabrigados. Outra decisão importante envolve o planejamento eficiente dos equipamentos para desobstrução dos acessos, uma vez que estes recursos são escassos na fase de resposta imediata.

O Quadro de Engenheiros Militares do EB também atuou no novo mapeamento da Região Serrana imediatamente após o desastre, com a finalidade de mostrar o melhor acesso às áreas que ainda estavam isoladas, facilitando assim o trabalho de resgate das vítimas. Esta medida também facilitou a indicação dos locais que podem ser ocupados, de modo a evitar novas construções em áreas indevidas. 


\subsection{Saúde e socorros médicos}

Os hospitais de campanha das Forças Armadas Brasileiras têm sido a solução no atendimento a vítimas de desastres no Brasil, especialmente em casos de enchentes e deslizamentos, tal como o evento analisado. Estes hospitais foram essenciais para a redução do tempo de espera para o atendimento às vítimas do desastre, dada a destruição de parcela dos recursos locais.

Os hospitais de campanha oferecem atendimentos ambulatoriais em especialidades de clínica médica, ortopedia, pediatria, ginecologia e odontologia. Contam com centros cirúrgicos, raios- $\mathrm{X}$, laboratórios e leitos para curtos períodos de recuperação. Em casos em que fosse necessária a transferência de pacientes, era necessária a evacuação aero médica. Segundo levantamentos do Hospital de Campanha da Marinha Brasileira, dos atendimentos realizados nas primeiras setenta e duas horas após o desastre, $75 \%$ são casos clínicos, $20 \%$ ortopédicos e $5 \%$ psiquiátricos.

Observou-se que, depois de traumas físicos, fraturas e lesões, a maioria dos atendimentos envolvia pacientes com problemas psicossomáticos, causados por sequelas emocionais.

Também foi essencial o trabalho médico no controle da leptospirose, doença à qual a população fica vulnerável em casos de enchentes e inundações. Outro ponto importante para a manutenção do bom estado sanitário envolveu a remoção e sepultamento de cadáveres. O Exército Brasileiro apoiou a execução dessas atividades, e ainda prestou assistência religiosa com o capelão militar. A partir da experiência neste processo, foi identificada a dificuldade na estimativa dos itens críticos para o atendimento das vítimas, uma vez que não há levantamentos sobre os tipos de acidentes e traumatismos mais comuns nestes tipos de desastres. Ainda, foram enfrentadas dificuldades devido a carências no suprimento de medicamentos e na doação de sangue. Logo, a principal decisão tomada se refere à alocação de recursos, como médicos e remédios, para maximizar o atendimento.

\section{4. Água, saneamento e higiene}

Segundo dados da Cruz Vermelha Internacional, a demanda diária de água potável por pessoa em casos de desastres varia entre quatro e cinco litros/pessoa/dia, incluindo nesta estimativa o consumo do recurso por hospitais e clínicas (MARTINEZ et al., 2010). A população das cidades atingidas pelo desastre da região serrana é de aproximadamente 700 mil habitantes, sendo que 30 mil vítimas ficaram desabrigadas. Deste modo, foi necessário um mínimo de 150 mil litros de água diários para atender a demanda dos desabrigados/desalojados pelo desastre. 
A água é um item crítico para a sobrevivência, de modo que o suprimento deste recurso foi tratado como uma prioridade nos primeiros dias após o desastre. Empresas e população do Rio de Janeiro doaram grande quantidade de água mineral atendendo à solicitação dos órgãos de assistência às vítimas com grande divulgação através da mídia (televisão e rádio). $\mathrm{O}$ Exército Brasileiro participou do tratamento e da distribuição de água em locais de difícil acesso. Devido à elevada demanda de suprimento de água e ao acesso restrito a locais isolados, foi observada a dificuldade em priorizar a distribuição de água na fase de resposta imediata e em como estabelecer o sistema de distribuição de água de modo a atender a maior demanda possível.

\subsection{Alimentos}

Em situações de grandes catástrofes, duas considerações precisam ser observadas quanto à alimentação: (i) o atendimento a população impactada; e (ii) o atendimento ao conjunto de pessoas, voluntários e profissionais que prestam os serviços à população. Especificamente, no caso do Exército, eram servidas 868 refeições por dia para alimentar a tropa empregada para prestar apoio em Teresópolis.

Neste aspecto, houve necessidade de controlar a distribuição de alimentos com intuito de atender todo o contingente. A distribuição de alimentos em casos de operações humanitárias é um problema de fluxo de rede com múltiplos modais e múltiplas commodities, cujo objetivo consiste em maximizar o atendimento da demanda à medida que o tempo de resposta é minimizado (MARTINEZ et al., 2010). Ressalta-se que o problema se torna ainda mais complexo porque, em alguns segmentos da rede viária, o trânsito está impedido devido a deslizamentos ou inundações.

É necessário um reabastecimento constante dos itens de alimentação, porém há uma carência de estudos de previsão da demanda dos suprimentos necessários. Também no aspecto de alimentação, houve uma resposta imediata de empresas e da população do Rio de Janeiro, mas foram enfrentados problemas de armazenagem destas doações. As principais decisões a serem tomadas com relação à distribuição de alimentos se referem a: (i) transporte das doações até a região do desastre; (ii) locais para armazenagem dos suprimentos; (iii) quantidade e localização dos pontos de distribuição de alimentos.

\subsection{Abrigos e suprimentos não relacionados à alimentação}

No total, foi estimado um número de 30 mil vítimas desabrigadas pelo desastre. A população desalojada foi inicialmente distribuída em escolas e galpões em condições 
de recebê-la. Os abrigos foram necessários para atender a população que precisava de atendimento médico. As vítimas também foram alojadas em barracas semelhantes às utilizadas no terremoto do Haiti e no tsunami na Indonésia (LAURIANO, 2011). As barracas, com capacidade para dez pessoas, possuem equipamentos de sobrevivência, fogareiros, talheres, panelas, pratos, cobertores, purificador e armazenador de água.

Com relação a suprimentos não relacionados à alimentação, observou-se excesso de doações de casacos, calças e camisas. Porém, houve a carência de doações de roupas de cama, toalhas e roupas íntimas $(O$ EXÉRCITO, 2011). Destaca-se a dificuldade em estimar as necessidades, de modo que era necessária a reposição constante dos itens, segundo a evolução dos fatos. Também foram apontados obstáculos para transportar o material, armazená-lo e distribuí-lo, em razão da dificuldade observada em prever a demanda.

\section{Considerações finais}

$\mathrm{O}$ presente artigo procurou discutir o conceito de logística humanitária a partir de uma abordagem teórica e prática, ressaltando a importância de sua efetiva aplicação em situações de desastre natural.

A análise sobre a atuação do Exército Brasileiro no socorro às vítimas da catástrofe ocorrida em janeiro de 2011 na região serrana do estado do Rio de Janeiro, enquanto parte executora de diversas ações logísticas revela, de um lado, o valor que um bom planejamento e organização para o atendimento a situações emergenciais representa, e de outro, as dificuldades em se trabalhar em situações de demanda imprevista, típicas de grandes catástrofes.

A logística humanitária se revela uma ferramenta decisiva para o melhor atendimento às vítimas de catástrofes, organizando o fluxo de informações e recursos materiais de maneira que o atendimento possa ocorrer com agilidade e eficácia, e seu estudo poderá trazer importantes contribuições para a sociedade.

\section{Referências}

BALCIK, B.; BEAMON, B; KREJCI, C.; MURAMATSU, K. E RAMIREZ, M.

Coordination in humanitarian relief chains: Practices, challenges and opportunities.

International Journal of Production Economics. v. 126, 2010. p.22-34.

BEAMON, B. E KOTLEBA, S. Inventory modeling for complex emergencies in humanitarian relief operations. International Journal of Logistics: Research and Applications, v. 9, n. 1, 2006. p. 1-18.

BLECKEN, A. Supply chain process modeling for humanitarian organizations. International Journal of Physical Distribution \& Logistics Management, v. 40, n. 8/9, 2010. p. 675-692. 
CAMPANATO, V. Um ciclo de calamidades precisa ser interrompido. Revista do CREA/RJ, v. 86, Janeiro/Março de 2011.

ERTEM, M., BUYURGAN, N. E ROSSETTI, M. Multiple-buyer procurement auctions framework for humanitarian supply chain management. International Journal of Physical Distribution \& Logistics Management, v. 40, n. 3, 2010. p. 202-227.

FERNANDES, C. W. N. O enfoque da logística humanitária na localização de uma central de inteligência e suporte para situações emergenciais e no desenvolvimento de uma rede dinâmica. 2010. Tese de doutorado. Depto de Engenharia de Produção, UFSC. Florianópolis, SC. 2010

GONÇALVES, D.B. Os Impactos no Meio Ambiente. In: XIII Workshop - Impactos da Evolução do Setor Sucroalcooleiro, 2008, Campinas. anais, 2008.

KOVACS, G. E SPENS, K. Identifying challenges in humanitarian logistics.

International Journal of Physical Distribution \& Logistics Management, v. 39, n. 6, 2009. p. 506-528.

LAURIANO, C. Tendas como as usadas após tsunami abrigarão vítimas de chuvas no Rio de janeiro. O Globo [online], Rio de Janeiro, 17 jan. 2011. Chuvas no Rio de Janeiro.

Disponível em : <http://g1.globo.com/rio-dejaneiro/chuvas-no-rj/noticia/2011/01/tendascomo-usadas-apos-tsunamiabrigarao-vitimasda-chuva-no-rj.html > Acesso em 1 de junho de 2011.

MARTINEZ, A.; STAPLETON, O. VAN WASSENHOVE, L. Using OR to Support Humanitarian Operations: Learning from the Haiti Earthquake. INSEAD Working Paper. 2010.
O EXÉRCITO da solidariedade em ação na Região Serrana. Revista Veja [online], Rio de Janeiro, 18 janeiro 2011. Disponível: http://veja.abril.com.br/blog/vejaacompanha/tag/teresopolis/page/2/ > Acesso em 27 de maio 2011.

ONU: desastre naturais atingem 7,5 milhões no Brasil. O Último Segundo [online], 25 janeiro 2011. Brasil. Disponível em: <http://ultimosegundo.ig.com.br/brasil/onu + desastres+naturais+atingem $+75+$ milhoes+no+ brasil/n1237967836366.html > Acesso em 1 maio de 2011.

THENÓRIO, I. Mapeamento é desafio para alertar catástrofes, diz pesquisador. O Globo [online], Rio de Janeiro, 20 jan. 2011. Ciência e Saúde. Disponível em: <http://g1.globo.com/cienciaesaude/noticia/2011/01/mapeamento-e-desafiopara-alertar-sobre-catastrofes-diz-

pesquisador.html> Acesso em 1 de junho de 2011.

THOMAS, A. e KOPCZAK, L.R. Life-saving supply chains - challenges and the path forward. In: Lee, H.L.and Lee, C.-Y. (Eds), Building Supply Chain Excellence in Emerging Economies. Springer, New York. 2007.

TRB- Transportation Research Board. A Guide to Planning Resources on Transportation and Hazards. 2010. Disponível em <http://www.trb.org/Publications/Public/Blurbs /162332.aspx>, Acesso em 20 de maio de 2011. 